\title{
A REVIEW: MONITORING AND SAFETY OF PILGRIMS USING STAMPEDE DETECTION AND PILGRIM TRACKING
}

\author{
S. K. Shah ${ }^{1}$, Sharley Kulkarni ${ }^{2}$ \\ ${ }^{1} H O D$ PG, STES'S Smt. Kashibai Navale College of Engineering, Pune, Maharashtra, India \\ ${ }^{2} P G$ Student, E\&TC, STES's Smt. Kashibai Navale College of Engineering, Pune, Maharashtra, India
}

\begin{abstract}
At present, there are so many problems regarding the crowd control, medical emergencies, security issues, identification and tracking of the pilgrims in the holy areas. Especially during pilgrimage, the pilgrimage authority finds it difficult to manage the situation. Thus, in order to identify, track and monitor pilgrims a system is needed. In this system camera is used to monitor continuously and to find high density with the help of image processing. The communication with base station is done through GSM. As soon as pre-stamped starts, people around pre-stamped, camera detect the picture and compare the density and message is send to the police if very high density is found. This embedded system is divided into two parts stamped detection and pilgrim monitoring unit. In Stamped detection unit continuous monitoring of the stamped scenario using Image processing via MATLAB software is implemented. The MATLAB software continuously takes snapshot at every 10 seconds and will analyse the scenario for stamped threshold. If the people gathered in a particular area are more than the threshold then stamped warning is sent to ARM microcontroller via RS232 and simultaneously we send an indication to the Pilgrim unit via GSM. In Pilgrim monitoring unit continuous monitoring of the status of pilgrim using the health parameters of pilgrim and sending the GPS Co-ordinates along with the health parameters is initiated.
\end{abstract}

Keywords: Matlab; ARM; GSM; GPS

\section{INTRODUCTION}

Pilgrimage has a great significance in India. Each pilgrimage session attracts a huge crowd. Usually the pilgrims move simultaneously in a large group. Getting lost in crowd is very common. Finding the lost person among thousands of pilgrims causes a lot of trouble for his relatives and the authorities. Such a setup poses a real challenge to the authorities in managing the crowd, and tracking/identifying People. What makes it even more challenging is that all pilgrims move at the same times and to the same places. While such events are a unique spiritual experience for all pilgrims, it poses major challenges of all sorts to the authorities responsible for facilitating this annual event. The following are some of the common difficulties faced by the pilgrims and the authorities like Identification of pilgrims (lost, dead, or injured), Medical emergencies, Guiding lost pilgrims to their respective camps, Congestion management.

The respective authorities of the holy areas are facing many problems. But they are unable to provide those facilities in a full fledge manner. However, providing a solution to solve the problems completely is impossible. For such a scenario, there is a need for a robust tracking system for pilgrims. Passive and active RFID systems have been tested in the past with limited success [1]. Other approach was using WSN to track the pilgrim [2]. Thus, the idea of using GSM/ZigBee for tracking pilgrims is initiated. As the elderly people participating in such events health related issues of them arises. Those have health problems may arise in severe situations, especially in large crowds and congestion, causing death in some cases. Even before efforts in health services by local and international authorities, unfortunately, it is difficult to monitor and intervene in time to save lives. Numerous existing applications using GSM are focused just on the identification of pilgrims and listing their information. Others focus only on the localization of pilgrims lost.

Continuous monitoring of crowd is the need of any religious expenditure. Surveying of large crowds can be done through processing the image captured of pilgrims in sacred areas. The stampede conditions starts building pilgrims can be made aware of such dangerous beforehand. Monitoring of pilgrim health can be done by using various sensors [1]. The real time location of a person can be tracked by using the GPS (Global Positioning System) and GSM (Global System for Mobile communication) technology.

\section{RELATED WORK}

Tracking and monitoring the pilgrims in a crowded area is difficult task. The pilgrims and the authorities faced the problems like locating pilgrims, medical emergencies, guiding lost pilgrims, congestion management. Various technologies used for tracking and monitoring pilgrims and some technologies consist of fusion.

\subsection{Technologies used in Tracking and Monitoring}

\section{Pilgrims}

Various systems have been designed by the researchers using GPS of Smartphone, RFID, standalone GPS with 
wireless communication for tracking the pilgrims. Some designs have other communication protocols for detecting the presence of people in an area like Bluetooth and $\mathrm{Wi}-\mathrm{Fi}$ not specifically tracking pilgrims. But they can be customized to track pilgrims.

\subsubsection{Smart Phone GPS}

M Mohandes proposed a system for the tracking and identification of pilgrims in the Holy areas, in MakkahSaudi Arabia, during Hajj [1]. The system consisted of $3.5 \mathrm{G}$ network which covered the area by several service providers. $3.5 \mathrm{G}$ is a grouping of disparate mobile telephony and data technologies designed to provide better performance than $3 \mathrm{G}$ systems, as an interim step towards deployment of full $4 \mathrm{G}$ capability. By request or periodically the UID (Unique Identification number) is send to the server. A server maps the latitude and longitude information on a Google map or any geographical information system. If the Internet connection is lost the mobile phone stores the location information in its memory until the Internet connection is restored, then it sends all stored location information and clears this information from memory. The developed system can be used to track a specific pilgrim. Alternatively any pilgrim can request emergency help using the same system. The developed system works in coordination with an RFID identification system.

T. Mantoro proposed a system called, Hajji Locator, which had been designed to track the pilgrims during Hajji. The system uses the GPS built in Smartphone. The data transfer is done preferably using $\mathrm{Wi}-\mathrm{Fi}$ or $3 \mathrm{G}$, but the system can also be used to send the data in emergency situations. A pilgrim is required to install an app on his Smartphone, which provides several features and sends his location to the server [2].

The frequency of location update is decided carefully as it affects the power consumption and the network bandwidth. The location is updated after a certain time period or after the user had moved a certain distance. Choosing time based method to update the location guaranteed that we had the updated location after a particular duration. But if a user is stationary or he is moving slowly, then the frequent location updates would be redundant and would drain the battery and increase the network traffic. The location based method, continuously kept the track of the current position and the last sent position. If the distance between current position and the last sent location is greater than a threshold value sent to the current position and stored it as last seen location. If a user moved very fast then distance based method would update more frequently.

The server used PHP and MySQL; Authorities could be logged in into the web server using a browser to get the position of pilgrims which is displayed in either a tabular form or Google maps. The proposed system concluded that the distances based update is recommended for tracking pilgrims [2].
Also an option is provided to the user to send his location manually. The accuracy in open or semi-opened to places is fine, but sometimes the server did not receive any data due to unavailability of a location fix. In some case, the location obtained from GPS is very far from user actual location. Using previous location of a user, these erroneous locations were adjusted and corrected.

\subsubsection{Integrated Mobile with RFID Systems}

Mitchell et al. proposed a system designed for tracking the pilgrims during Hajj. In this system, an RFID tag is given to each pilgrim. Pilgrims having Smartphone with GPS used location based services by installing an app. These services included location family members or friends, requesting urgent help, a map of important locations. To be able to transmit the current position, the app must be running in the phone. RFID readers were also installed in different regions to scan the tags.

The control center provides features like visualizing he location of all the pilgrims on a map searching for pilgrims based on several criteria like region, age, etc. sending notification to the mobile device, maintaining the database of places like hospitals, location history and personal information about pilgrims. Smartphone use web services and the RFID readers use middleware software to interface with the control centre [3].

These systems faced problems with RFID tags and readers. The read range of the RFID reader is low; it is also affected by the environmental factors. The signal between thr RFID tag and the reader is getting blocked by the tag holders own body, also the read range affected by angle with reader. So they decided to not have a wristband RFID tags.

\subsubsection{Wireless Sensor Network}

M. Haleem et al. proposed a system each pilgrim is given a sensor unit which contained a GPS for getting the current location of the pilgrim, a microcontroller which executes the program to send the location to fixed sensor units, a battery to power this matchbox sized sensor unit. It also includes ZigBee radio which is used to transfer the data to a network of fixed master units deployed in the region of interest. The tracked mobile units can be much more than fixed sensing nodes. The designers of these systems had developed an RFID based systems in the past which provided only the identification of pilgrims. This system is designed to track pilgrims as well as identify them [4]. The wireless sensor nodes transmit their unique identification number, its current position as obtained from the GPS ant the time periodically or on request from tracking station. The data transfer takes place opportunistically using ad hoc network. The sensor units transmit the data using flooding protocol, so the same data is sent to all the nearby fixed sensors. This data is stored for some time and then multi hop routing is used to transfer this data. The system designed can tolerate failure of a few fixed sensor nodes. The positions of pilgrims were mapped onto Google map similar to other systems. 
For querying the location of a pilgrim, the server sends queries by using optimal route utilizing the last know location. The system also supports routing multiple queries in parallel. Battery powered wireless sensors units should have power efficient hardware and software. The data transfer should also be minimum. The increased frequency of updating the location affects power consumption and bandwidth, but it reduces the time taken to find a pilgrim [4].

\subsubsection{Bluetooth}

A number of class 2 Bluetooth devices connected to host machine were distributed in the area of interest. All of these host machines were networked together. This setup can be simply added to a Bluetooth dongle to a laptop or desktop. $\mathrm{X}$. W. Wong and Y. Kong proposed a central system which is connected to all these hosts and 1 a database which contains the information about the ID of device, the owner of device, etc [5]. This central system also maintains a model of the current positions of all the persons. This information is used to ask a host machine to scan for the device IDs of the persons who can be there. For example, if a person leaves one checkpoint, he will probably go to the next check point, so the next check point can be ordered to scan the ID of that person's device.

The system requires frequent connection and disconnection, so the connection time should be minimum. The connecting time depends on the clock synchronization between the devices. Once a host knows the clock difference between the attached Bluetooth devices clock and a Bluetooth devices clock, it can distribute that information to other hosts. To work this properly, the hosts must have their clocks synchronized. It can tolerate an error of upto 10.24 seconds. The time offset between the hosts as well as their Bluetooth devices clock offset needs to be synchronized. This technique enables us to get an expected connection time of 0.64 seconds.

\section{$2.15 \mathrm{Wi}-\mathrm{Fi}$}

$\mathrm{X}$. Wang proposed a combine work of AGPS/Wi-Fi and cell ID to optimize the performance of tracking system. Assisted Global Positioning Systems (AGPS) is available on most cell phones and can be used to precisely locate a user, but has several drawbacks. Although AGPS can greatly reduce the power consumption .most cell phones will run out of battery in a matter of hours if AGPS location fixes were performed frequently [6]. Cell Id positioning has less power consuming and is available both indoor and outdoor, but estimation of location is less accurate. Hence AGPS/WI FI system is proposed along with concept of mobility mapping. WIFI mainly used to detect the presence of person. The drawback of using Wi-Fi is that it mainly works well indoors.

\subsection{Fusion of Technologies used In Tracking and}

\section{Monitoring}

The technologies described in above section can be fused together to implement the tracking and monitoring of pilgrims along with crowd monitoring.

\subsubsection{RFID and "Smart Watch"}

M. Yamin et al. proposed the RFID technique to control and manage crowd. The whole area is installed with the surveillance systems, the managers were to continuously monitor the situation and take the preventive measures. The problem related to identification of pilgrims is solved. Whenever a stampede or a fire occurred, identifying badly mutilated bodies became a challenge. Another result of overcrowding is that thousands of pilgrims were disintegrated from their groups or from relatives for days or weeks (and some of them may never found). Reuniting the pilgrims with their groups may take considerable time due to poor identification mechanisms in place. In cases when pilgrims were lost and do not know their place of stay, there is no way offending their details, without seeking the help. If pilgrims do not return to their bases, there is absolutely no technology in place to track them. Hence the RFID and "smart watch" systems supported by the backend database would be able to track the missing persons, and find all the other details is proposed in [7]. As the biometric scans for each pilgrim would also be stored in the backend database, it would help identification in case the loss of the RFID tags and 'smart watch'. Once the people with health risks were identified, they can be monitored throughout the pilgrimage by the established RFID and "smart watch" system. The PDA can be linked with the "smart watch" system, by which the person can clearly see the situation and also the PDA can talk to the "smart watch" system to send the "SOS' signal to the central control station so that the medical aid can be organized on an urgent basis. Since the systems would know the exact location of the patient, there would be no delay in sending the medical help.

The power consumption is a significant limitation in the smart watch architecture; this is due primarily to the Graphical User Interfaces (GUI's) and Operating Systems (OS's) used. Smart watches which were not architecturally designed to conserve power, still have to be charged regularly in order to be used. The miniaturized screen on the smart watch can be seen as both an advantage and disadvantage. The problem of interference lies within the RFID system such as stationary-to-stationary readers, stationary-to-mobile readers, and mobile readers-to-mobile readers in denser environments because the RFID system uses the same frequency bands, there is a serious problem of spectrum congestion and interference between various types of networks. Interference problem also leads to misdetection of important tags and reading the same tags multiple times.

\subsubsection{RFID with ZigBee}

A. M. Nair and S. J. Daniel proposed RFID technology which describes the design and implementation of a system 
for tracking and monitoring the pilgrims. The transmitting section consisted of RFID reader, microcontroller and ZigBee transceiver. The transmitting unit sent the current location and unique ID to the server unit using ZigBee transceiver. The receiver unit consists of ZigBee transceiver, external EEPROM unit and microcontroller unit. The received location and unique ID will be stored into the external EEPROM periodically. A heart beat sensing unit is connected to the transmitting section to monitor the medical condition in case of emergency. The pilgrims were tracked and the medical assistance can be sent in no time [8].

\subsection{GSM/GPS Technology and Smartphone}

\section{Application}

S. Lee et al. proposed a system for tracking the vehicle which is similar to that of tracking pilgrims. The system designed is efficient and can be implemented for tracking the movement of any equipped vehicle from any location at any time. The proposed system can make good use of a popular technology that combines a Smartphone application with a microcontroller. The system is easy to make and is inexpensive to users. The design in vehicle device worked using Global Positioning System (GPS) and Global system for mobile communication / General Packet Radio Service (GSM/GPRS) technology that is one of the most common ways for vehicle tracking. The device is embedded inside a vehicle whose position is to be determined and is tracked in real-time [9]. A microcontroller is used to control the GPS and GSM/GPRS modules. The vehicle tracking system used the GPS module to get geographic coordinates at regular time intervals. The GSM/GPRS module is used to transmit and update the vehicle location to a database. A Smartphone application is also developed for continuously monitoring the vehicle location. The Google Maps API is used to display the vehicle on the map in the Smartphone application. Thus, users will be able to continuously monitor a moving vehicle on demand using the Smartphone application and determine the estimated distance and time for the vehicle to arrive at a given destination.

\section{PROPOSED METHODOLOGY}

The proposed block diagram is shown is figure 1. It consists of ARM controller, GSM/GPS module, Android cell phone, SD card, loudspeaker, LCD display, pulse rate monitoring sensor and temperature sensor. Figure 2 shows the server block diagram. It consists of PC server and GSM module.

\section{1 Working}

Stampede detection and pilgrim tracking is implemented on same controller. Stampede detection and pilgrim tracking can be considered as two separate units.

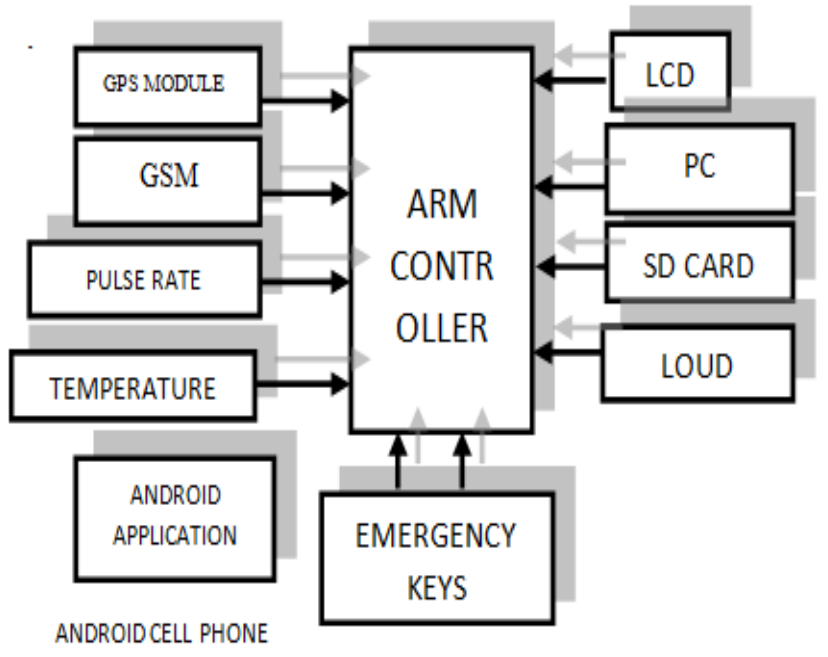

Fig 1: Block Diagram of Stampede Detection and Pilgrim Tracking With Health Monitoring

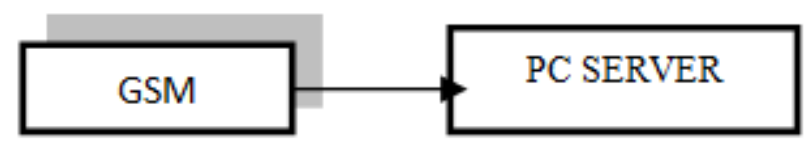

Fig 2: Block Diagram Monitoring System

\subsection{Stampede Detection Unit}

This unit comprises of SD card, loudspeaker, Webcam, PC and LCD. Continuous monitoring of the stamped scenario is done using Image processing via MATLAB software. Webcam will capture the live scenario of the target area. The stampede threshold will be set to monitor the different stampede like conditions. The MATLAB s/w will continuously take snapshot at every 10 seconds and will analyse the scenario for stamped threshold. If the people gathered in a particular area are more than the threshold then stamped warning is sent to $\mu \mathrm{C}$ via rs 232 and simultaneously announcements are done through loudspeaker from previously saved video clips.

1. Webcam: It is used to do the video surveillance of the pilgrim area continuously.

2. PC: the surveillance video is inputted to PC. Snapshots are taken and are processed through MATLAB software.

3. Image Processing: the images are processed to get the required format. The processed image is then compared with the threshold set previously. If the threshold value found is high then warning is sent to $\mu \mathrm{C}$.

4. Microcontroller: the controller then accesses the SD card and data is out according to the particular threshold value set for a particular voice clip though loudspeaker.

\subsection{Pilgrim Detection Unit}

Pilgrim detection unit comprises of GPS module, GSM module, and pulse rate monitor and temperature sensor. Pulse rate and temperature sensor monitor the status of pilgrims using the health parameters of pilgrim 
continuously. GPS co-ordinates along with health parameters of pilgrims are sent through GSM.

1. GPS: Global Positioning System finds the location of pilgrim by considering the longitude and latitude. The co-ordinates are sent to other GPS module on the server side.

2. GSM: Global System for Mobile Communication is used to send the data related to pilgrims location and health.

3. Pulse rate sensor and temperature sensor: These sensors sense the pulse rate and body temperature of the pilgrim.

\subsection{Monitoring System}

In monitoring system Visual basic software will be used in personal computer as shown in figure 3.2. Programmers can not only create simple GUI applications, but to also develop complex applications. Display windows are created using drag-and-drop techniques. Pulse rate and temperature send by pilgrim unit can be display. The location of pilgrim is also displayed using Google Map. ARM controller in system will be programmed using Keil UV3 of Embedded C programming.

\section{CONCLUSION}

The proposed architecture uses the single GPS /GSM modem which provide exact location of pilgrims and high speed communication instead of using separate GSM and GPS modems.Stampede conditions can be detected beforehand to take necessary action in time to prevent it and thus provide security to pilgrims.Apart from tracking and detecting stampede detection, the proposed system has a great advantage. It presents an option for a pilgrim in case of medical emergency.The developed system automatically monitors the body conditions of the pilgrim which helps in getting medical aid in no time. Thus the system can satisfy the need of pilgrim without any objection.

\section{REFERENCES}

[1] Mohandes $M$ "Pilgrim tracking and identification using the mobile phone" Consumer Electronics (ISCE), 2011 IEEE 15th International Symposium, 2011

[2] Mantoro, T. "Hajj Locator: A Hajj pilgrimage tracking framework in crowded ubiquitous environment" Multimedia Computing and Systems (ICMCS), 2011 International Conference, April 2011

[3] Mitchell, R.O. ; Dept. of Comput. Sci. \& Eng., Univ. of Yanbu, Yanbu, Saudi Arabia ; Rashid, H. ; Dawood, F. ; AlKhalidi, A. "Hajj crowd management and navigation system: People tracking and location based services via integrated mobile and RFID systems" Computer Applications Technology (ICCAT), 2013 International Conference, 2013

[4] Mohamed Mohandes, Mohamed Haleem, Mohamed Deriche, and Kaviarasu Balakrishnan "Wireless Sensor Networks for Pilgrims Tracking" IEEE
Embedded Systems Letters, Vol. 4, No. 4, December 2012

[5] Barahim, M.Z. ; Univ. of Mauritius Reduit, Reduit ; Doomun, M.R. ; Joomun, N. Low-Cost Bluetooth Mobile Positioning for Location-based Application Internet, 2007. ICI 2007. 3rd IEEE/IFIP International Conference in Central Asia, 2007

[6] Xiaoli Wang; Wong, A.K.-S. ; Yongpmg Kong "Mobility tracking using GPS, Wi-Fi and Cell ID" Information Networking (ICOIN), 2012 International Conference on 2012

[7] Mohammad Yamin, Masoud Mohammadian, Xu Huang, Dharmendra Sharma "RFID Technology and Crowded Event Management" CIMCA 2008, IAWTIC 2008, and ISE 2008

[8] Anju .M. Nair, S. Joshua Daniel “ Design of Wireless Sensor Networks for Pilgrims Tracking and Monitoring "International Journal of Innovations in Scientific Engineering Research(IJISER), Vol 1 Issue 2, Feb 2014

[9] SeokJu Lee, Girma Tewolde, Jaerock Kwon "Design and Implementation of Vehicle Tracking System Using GPS/GSM/GPRS Technology and Smartphone Application" IEEE World Forum on Internet of Things (WF-IoT) , 2014 